\title{
Gradhiva
}

GRADHI

Revue d'anthropologie et d'histoire des arts

$33 \mid 2022$

Wampum : les perles de la diplomatie

\section{Wampum. Perles de diplomatie en Nouvelle-France}

\section{Paz Núñez-Regueiro et Nikolaus Stolle}

\section{OpenEdition}

\section{Journals}

Édition électronique

URL : https://journals.openedition.org/gradhiva/5877

DOI : 10.4000/gradhiva.5877

ISSN : 1760-849X

Traduction(s) :

Wampum. Beads of Diplomacy in New France - URL : https://journals.openedition.org/gradhiva/6173 [en]

\section{Éditeur}

Musée du quai Branly Jacques Chirac

Édition imprimée

Date de publication : 2 février 2022

Pagination : 6-21

ISBN : 978-2-35744-133-0

ISSN : 0764-8928

Référence électronique

Paz Núñez-Regueiro et Nikolaus Stolle, "Wampum. Perles de diplomatie en Nouvelle-France », Gradhiva [En ligne], 33 | 2022, mis en ligne le 02 février 2022, consulté le 14 septembre 2022. URL http://journals.openedition.org/gradhiva/5877 ; DOI : https://doi.org/10.4000/gradhiva.5877 



\section{Wampum}

Perles de diplomatie

en Nouvelle-France

\section{Paz Núñez-Regueiro et Nikolaus Stolle}


1. Ce terme d'origine algonquienne désigne à l'époque une perle blanche lorsqu'il est employé au singulier, et un cordon blanc lorsqu'il est utilisé au plurie (Ceci 1989: 73).

2. Ceci est suggéré par les vestiges archéologiques (Ceci 1989) et les données linguistiques (Michelson 1991)

3. Décrites par Lescarbot comme «petits tuyaux de verr méle d'etain, ou de plomb» (1609: 740) et documentées par la découverte, en contexte archéologique, de tubes de verre noirs ou rouges, comprenant parfois des bandes colorées parallèles supplémentaires (Wray 2003 : 44; Stolle 2016: 265, table 5, $\mathrm{n}^{\circ \mathrm{s}} 1$ et 2 )

4. Pour un récapitulatif des termes associés au wampum à la période de la colonisation française en Amérique du Nor se référer au tableau en annexe.

5. Innus de nos jours. Pour une meilleure concordance avec les sources historiques, nous employons dans le texte les désignations européennes de l'époque.

6. Aujourd'hui Lenni Lenape.

7. Aujourd'hui Haudenosaunee et Hurons-Wendat

respectivement.

8. Foster 1996: 98-100, 105-109. Des nations des familles linguistiques muskogienne et siouanne habitent cette même région: si elles adoptent ponctuellement le wampum à la période coloniale, celui-ci n'acquiert cependant jamais n'acquiert cependant jamais la valeur universelle qu'il revêt
pour les groupes algonquiens pour les groupes algonquiens
et iroquoiens (Foster 1996: 98-106, 109-110; Silverstein 1996: 124-126).

9. Hewitt 1902: 33-46; Black 1977: 141-151; Isaac 1977: 167-184; Feest 1998: 76 sq

10. Hale 1881: 10 sq. Pour plus d'informations sur la fondation de la Ligue iroquoise, voir: de la Ligue iroquoise, voir: Fenton 1998: 76 sq.

11. À titre d'exemple, la nomination des chefs est bien documentée pour les Penobscot au début du xIx siècle (Stolle 2016: 65 du xIx ${ }^{e}$ siècle (Stolle 2016 .
n. 104). Au xvIII ${ }^{e}$ siècle, l'attribution des noms en général a été documentée parles Frères moraves pour les Deleware Lenni Lenape (Stolle 2016: 66, n. 106).

12. En contexte iroquoien, les objets funéraires en wampum du XVII ${ }^{\mathrm{e}}$ siècle proviennent de sépultures d'individus de haut rang (Wray 2003): en contexte algonquien, ils ont été retrouvés dans des sites archéologiques de la région de Rhode Island (culture wampanoag) et sur le site de Lasanen dans le Michigan (culture outaouais). Voir:Stolle 2016: 44
À l'orée du XVII siècle en Amérique du Nord, de petites perles cylindriques en coquillage marin dénommées wampum ${ }^{1}$ commencent à être employées comme objets d'échange entre Amérindiens et Européens. On se sert du wampum chez les nations autochtones, dans un cadre social, politique, ainsi que comme objet de parure. Il est déjà un objet d'échange de grande valeur parmi les nations nord-amérindiennes, avant même leur contact avec les Européens ${ }^{2}$. Les perles employées à ces périodes anciennes, manufacturées à partir d'un coquillage blanc qui se développe le lonǵ de la côte atlantique nord jusqu'au golfe du Mexique, sont rares et restent l'apanage des personnes au statut important.

Parmi les premiers produits de traite d'origine européenne, les perles ǵlobulaires et tubulaires de verre de différentes couleurs ( «rassades») correspondent en taille et en forme à ces perles antérieures (Turgeon 2001: 72). Leur introduction offre à la population locale la possibilité d'enfiler et de tisser les perles blanches traditionnelles en combinaison avec des perles de verre colorées, principalement noires et rouges ${ }^{3}$, afin de créer des «ficelles» (ou «branches») et des «colliers» (plus rarement nommés «ceintures» par les Français [Vachon 1970 4]) aux motifs décoratifs variés. Par ailleurs, l'introduction d'outils métalliques en Amérique du Nord ouvre la voie à la production de perles de couleur violette, manufacturées à partir de la lèvre violacée du Mercenaria mercenaria, un bivalve dont la coquille est particulièrement épaisse et dure à perforer.

Ces innovations ont lieu dès l'origine de l'histoire coloniale de la France dans la région, et les colons français y contribuent de manière signnificative. Au début du XVII ${ }^{\mathrm{e}}$ siècle, les Français apprennent à se comporter «selon la coutume du pays» (Champlain 1603 : 3R) pour établir une politique d'alliances fondée sur l'amitié avec les nations autochtones, à l'instar de celle que François Gravé du Pont et Samuel de Champlain ont entreprise à la pointe des Alouettes, au confluent du fleuve Saint-Laurent et de la rivière Saǵuenay, avec un groupe alors désigné par les Français comme «Montaǵnais ${ }^{5}$ », rencontré le 27 mai 1603. Les Français y participent en effet aux harangues dans la cabane du chef Anadabijou et fument le pétun (tabac) avec leurs interlocuteurs (Ibid. : 3R-4R). Champlain, nommé premier commandant de la Nouvelle-France (1612-1629), évoque dans ses écrits l'importance symbolique des présents lors des rencontres entre nations autochtones, notamment du wampum, désiǵné sous le terme de «porcelaine» par les Français. Dans toute la vallée du Saint-Laurent, on trouve le wampum tant dans l'ornementation personnelle que dans les relations sociales, religieuses, commerciales et diplomatiques.

\section{LES CONTEXTES RELATIONNELS DU WAMPUM}

Le wampum relève en effet de tous les domaines de la vie, qu'il soit porté en parure, présenté en gage d'amitié et d'alliance, ou pour inviter à la guerre.
Cette conception du wampum partagée par les nations des groupes linguistiques algonquien et iroquoien, établies dans l'Est nord-amérindien, s'étend, d'est en ouest, de la côte atlantique jusqu'à la confluence du Missouri et du Mississippi, et du nord au sud, de la vallée du Saint-Laurent au sud des Appalaches (voir carte 1). Les Algonquins de la côte (Pequot, Delaware $^{\boldsymbol{6}}$, Susquehannock), comme les Iroquoiens du territoire des États actuels de l'Ontario et de New York (Iroquois et Hurons ${ }^{7}$ ), vivent dans des villages fortifiés composés de plusieurs maisons longues abritant un ensemble de familles. Les groupes de parenté se distinguent par un ancêtre commun et constituent des clans. Leur subsistance dépend de la chasse, de la pêche et de la culture de denrées telles que le maïs, le haricot et la courge. Les Algonquins septentrionaux et occidentaux (Abénakis, Montaǵnais, Outaouais, Ojibwa), qui pour la plupart ne cultivent pas la terre, habitent le territoire en groupes plus réduits, chaque cercle familial vivant dans des habitations coniques ou en forme de dôme appelées wigwams. À la saison estivale, ces familles se rassemblent en groupes élarǵis pour participer à des chasses et cérémonies communautaires, ainsi qu'à des campaǵnes militaires ${ }^{8}$.

Dans les deux groupes linguistiques, les hommes acquièrent leur statut en fonction de leur succès à la chasse et à la guerre. Les exploits guerriers, tels que la prise de trophées ou la capture de prisonniers en remplacement de parents décédés, constituent des faits méritoires bien souvent conditionnés par la présentation de colliers de guerre de wampum, qui peuvent être acceptés ou refusés par un guerrier ou un chef d'expédition militaire ${ }^{9}$. Sur le plan politique en effet, l'offrande joue un rôle fondamental parmi les nations nord-amérindiennes, et le don de wampum revêt pour les Iroquois un rôle central dans le fonctionnement de leur Confédération. Le mythe de fondation de leur Ligue met en scène Dekanawidah, le Grand Pacificateur porteur d'un message pacifique, qui parvient à faire la paix avec le chef despote onondaga Tatodaho par l'offrande d'une branche de wampum blanc. Dès lors, les perles en coquillage blanc vont occuper une place centrale dans toutes les manifestations politiques des Cinq puis Six Nations iroquoises (Mohawk, Oneida, Onondaga, Cayugáa et Seneca, puis après 1722 Tuscarora $\left.{ }^{10}\right)$. Un tel lien historique entre les perles en coquillage et la structuration politique n'est pas vérifiable pour les Algonquiens au XVII siècle.

Les Iroquoiens du Nord (Iroquois et Hurons) et les Algonquiens de l'Est (à l'instar des Abénakis et des Delaware) incorporent tous la présentation et l'offrande de wampum dans divers rituels, telles la cérémonie d'imposition du nom (au cours de laquelle des ficelles enfilées de perles de wampum appelées «branches», symbolisant le nom dont un clan est propriétaire, sont offertes à l'individu ${ }^{11}$ ) ou la célébration des funérailles (qui inclut le don de colliers et de branches de wampum pour accompagner le défunt dans son voyage vers l'au-delà, vraisemblablement comme symbole de prestige ${ }^{12}$ ). 
fig. 1. Valves du bivalve utilisé pour la confection des perles wampum violettes,

première moitié du xvII siècle. Palourde (Mercenaria mercenaria), $11,2 \times 9,1 \times 3 \mathrm{~cm}$, inv. 71.1881.17.4.

Ancienne collection du Muséum national d'histoire naturelle, Paris ๔ musée du quai Branly-Jacques Chirac, photo Pauline Guyon. 


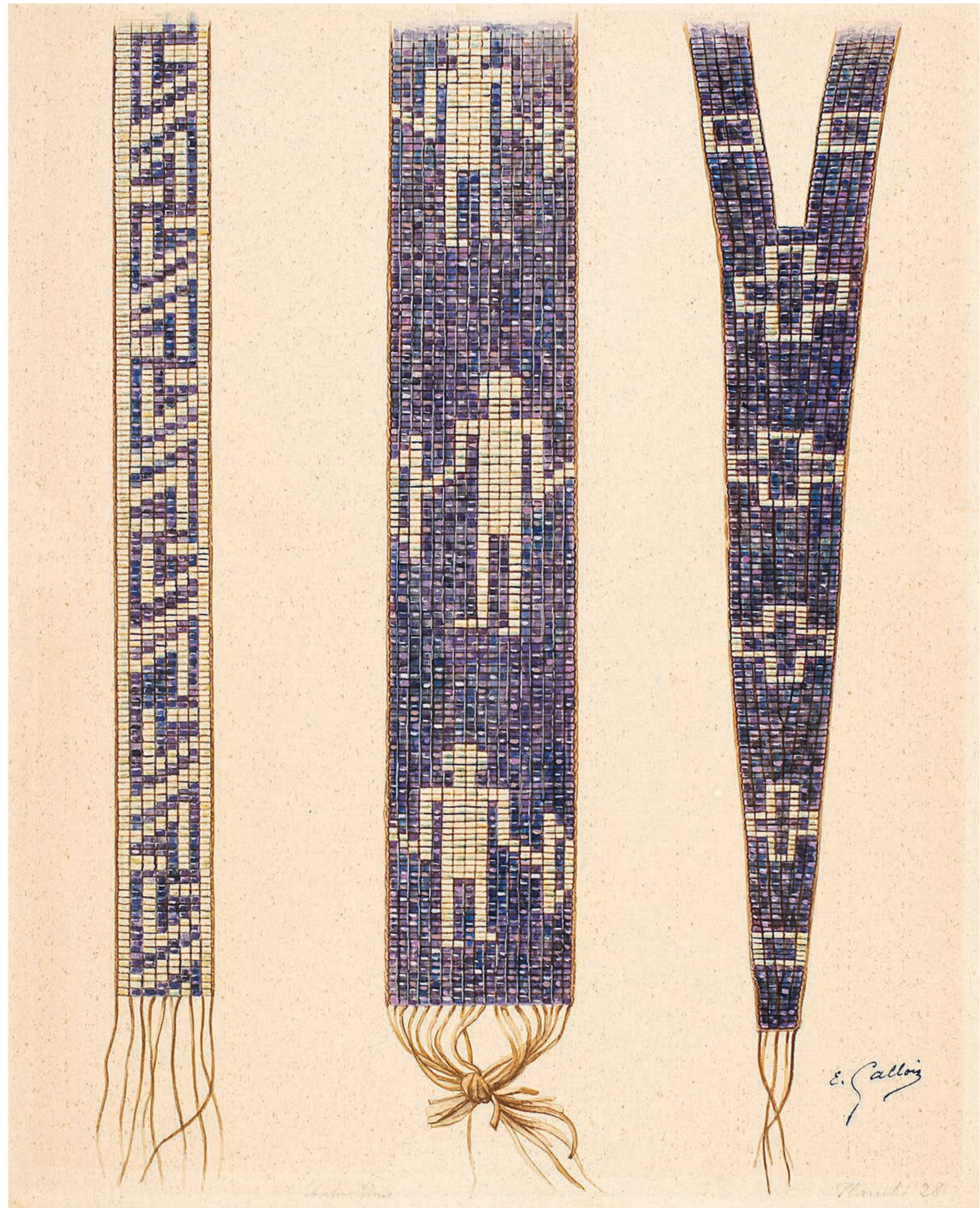

fig 2 Émile Gallois. États-Unis, planche 28 [planche illustrant trois colliers de wampum] vers 1945-1956. Dessin aquarellé sur papier $41 \times 33 \mathrm{~cm}$ (c) photo Thierry Ollivier. Musée du quai Branly-Jacques Chirac, acquisition en cours. Cette planche fait partie d'une édition présentant vraisemblablement les pièces les plus anciennes et prestigieuses du fonds américain conservé au musée d'Ethnographie du Trocadéro ou au musée de l'Homme, datant sans doute de 1933 et $1945-1956$. 
Ces valeurs attribuées au wampum, acceptées de manière généralisée en Amérique du Nord et basées sur la production chronophage des perles en coquillage, conduisent les puissances européennes installées dans la région à choisir ce matériau (perles et ficelles de wampum) comme substitut à la monnaie. Si la Nouvelle-France dispose d'une monnaie en argent, ce n'est pas le cas de la Nouvelle-Anǵleterre ni de la Nouvelle-Néerlande qui adoptent comme principaux substituts le wampum ou le tabac, commercialisés à un taux d'échange fixe dès le XVII ${ }^{\mathrm{e}}$ siècle. Les perles de wampum étant fabriquées sur la côte atlantique par les Algonquiens puis échangées contre des fourrures avec les Iroquoiens et les Algonquiens établis à l'intérieur des terres, c'est à bon escient que les Cinq Nations iroquoises puis les Européens (Anǵlais et Hollandais) choisissent de soumettre militairement ces nations: le contrôle de la production du wampum est la porte d'entrée privilégiée à la traite pelletière et aux réseaux d'échanges autochtones (Ceci 1993: 58 sq.).

Les Français, qui ont très tôt une très bonne compréhension des protocoles autochtones, adoptent le langage et vocabulaire diplomatiques, ainsi que la pratique de présentation de wampum ${ }^{13}$. Celle-ci est en effet devenue indispensable aux relations d'échange et d'alliance avec les nations amérindiennes:

\section{Les Sauvages de l'Amérique septentrio- nale ont été de tous tem [p]s dans l'usage de se servir de coliers tant pour les ornemens que pour traiter les affaires de leur nation; ces coliers sont si necessaires à ceux qui parlent d'affaires au nom des nations qu'on n'ajouteroit aucune foy a leurs paroles si préalablement ils ne presentoient a celuy avec lequel ils ont à traiter un colier qu'ils étendent devant luy; après le discours fini, le Sauvage auquel on s'adresse prend le colier et en remet un autre a la place pour faire sa reponse. \\ (BnF NAF 2550, Anonyme s. d. [vers 1725] : fo 24)}

Les colliers viennent sceller l'entente entre les parties, matérialisant en quelque sorte le discours qui accompagne leur présentation. Ils sont échangés entre individus ou représentants de nations souveraines, comme gage de sincérité et témoignnage de la parole donnée. Cette perspective européenne est sans doute trop restrictive et omet le rôle performatif de socialisation conféré au wampum dans les contextes relationnels où il est employé. Le collier offert «débouche les oreilles», «essuie les larmes», «nettoie le gossier», pour engager l'écoute, l'attention et la parole; il «débroussaille le chemin» pour «arriver au cœur» de son interlocuteur et l'«éclaire»; enfin, il contribue à bâtir «l'Arbre de Paix» entre les parties et à le préserver, «haut jusqu'au ciel», «inébranlable et à l'épreuve de tous les orages, tant les racines sont profondes dans la terre»(BnF NAF 2550, Anonyme s. d. [1726]). Ce langage hautement métaphorique, employé dans les conseils nationaux comme dans les rencontres entre nations, évoque la capacité du wampum à créer les conditions de l'écoute et du dialogue en agissant sur les sens et en transformant l'individu qui participe de ces échanges.

C'est leur compréhension du monde amérindien qui permet aux Français d'étendre leur influence sur un immense territoire: au gré de l'avancée de la colonisation vers l'ouest et dans la vallée du Mississippi, l'usage du wampum se diffuse le long du fleuve Missouri et jusqu'au golfe du Mexique ${ }^{14}$; l'iconographie que l'on trouve sur les wampums se complexifie et nombre de nations des Plaines centrales et méridionales intègrent l'usage de ficelles de perles en coquillage blanc dans leur répertoire diplomatique. Si ce sujet reste encore peu étudié, les sources suggèrent en effet qu'à partir du XVIII ${ }^{\mathrm{e}}$ siècle les Osage ${ }^{15}$, les Otoe, les Missouri et les Iowa ont recours aux ficelles de wampum à des fins diplomatiques, les fixant notamment aux calumets comme cela est documenté au moins pour les Iowa jusqu'en $1845^{16}$.

\section{LA PORCELAINE DES FRANÇAIS}

La Couronne de France opte pour une stratégie d'alliance visant à s'assurer le succès du commerce, en particulier la traite pelletière. Les Français vont ainsi s'insérer dans la géopolitique amérindienne telle une «nouvelle tribu» (Havard et Vidal 2019: 70 et 78), et se poser progressivement comme arbitres entre les nations amérindiennes alliées. Le succès de cette politique d'alliance débouche sur la «Grande Paix de Montréal » de 1701, qui vient mettre fin à un siècle de conflits désignés sous le terme de "guerres iroquoises » et pacifie un immense territoire allant de l'Acadie (Miramichi, Souriquois 17), à l'est, aux confins occidentaux et septentrionaux du lac Supérieur (Cris), à l'ouest, et du nord au sud, des sources de la rivière des Outaouais (Outaouais) au «pays des Illinois», situé à l'est au confluent du Missouri et du Mississippi (Kaskakia $\left.{ }^{18}\right)$. Les accords de Montréal réunissent trente-neuf nations d'Iroquoisie, d'Huronie et de l'Est (notamment Armouchiquois, Abénakis) et des Grands Lacs (Nipissing, Miami, Mesquakie, Sauk, Illinois notamment), venues siǵner un traité de paix avec les représentants de la Nouvelle-France sous l'impulsion de Louis-Hector de Callière, quatorzième gouverneur de la colonie (1698-1703) [Beaulieu et Viau 2001]. La présentation de colliers et de ficelles de wampum, accompaǵnant les paroles des représentants de chaque nation, occupe cette fois une place prééminente: dans ses quatre tomes de l'Histoire de l'Amérique septentrionale, Bacqueville de La Potherie accorde une place centrale à la reproduction des «paroles » ou harangues associées aux «colliers de porcelaine» échangés à cette occasion (Bacqueville de La Potherie 1722).

Le «Mémoire concernant les coliers de Porcelaine des Sauvages, leurs différens usages, et la matiere dont ils sont composés» (voir archive dans ce numéro p. 144) s'attarde longuement sur la manufacture des perles de wampum, documentée par une étude commandée à la Nouvelle York en 1725: les perles en coquillage blanc, préparées sur la pierre et percées avec une pointe de silex, sont lonǵues à produire (pas plus de vinǵt
13. Ce fait est souligné par différentes sources anglaises de l'époque, notamment sir William Johnson, fonctionnaire colonial et négociateur diplomatique pour la Couronne britannique pendant la guerre de Sept Ans, qui constate la méconnaissance du bon usage des colliers de wampum chez les Anglais, alors que les Français «in their time, always gave them [aux nations

amérindiennes] belts, rum and amerindiennes] belts, rum and
money, presents by which they renewed their peace annually» (Johnson 1951: 698).

14. En 1763 , des délégués onondaga informent sir William Johnson «que les Français se sont rendus parmi les

nombreuses nations d'Indiens du Sud-Est [en référence aux Cherokee et aux Choctaw] et de l'Ouest [Miami, Illinois et Kickapoo] leur offrant des colliers de wampum et des haches de guerres $\&$ les encourageant de toute leur forse de déposséder les Anglais de tous les postes dont ils ont pris possession aussi loin que Oswego» (Johnson 1951: 724) Ce phénomène est accentué par le déplacement forcé de nations à compter du début du xIX ${ }^{e}$ siècle vers le «territoire mexicain», aujourd'hui la région du Texas. Ainsi, I'usage de du Texas. Ainsi, l'usage de
«perles blanches» y est alors attesté notamment parmi les Cherokee, les Delaware, les Kickapoo et les Cutchatas (Mier y Terán 2000 [1828]: 77-78).

15. Ceux-ci sont dits faire la paix en employant le calumet de paix et les colliers (Cruzat 1909 [1777]).

16. Voir Nuñez-Regueiro et Stolle dans ce numéro; Keagle 2013: 222-225; Stolle 2016: 36 $242,249$.

17. Aujourd'hui Mi'kmaq/ Micmac.

18. Les Kaskaskias sont I'une des quatre tribus qui forment la nation des Illinois (Callender 1978: 673, 678-679). 
VEU்E DE QUEBEC

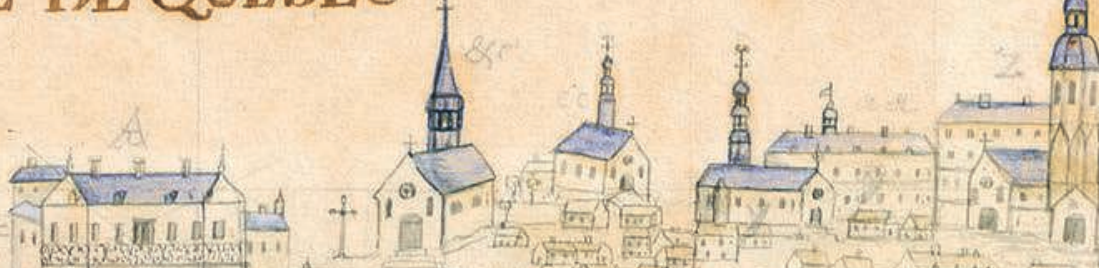

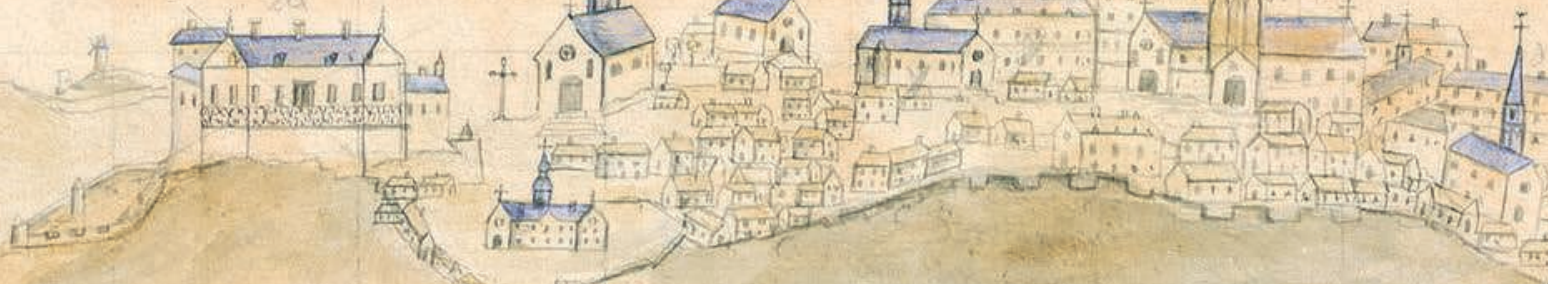

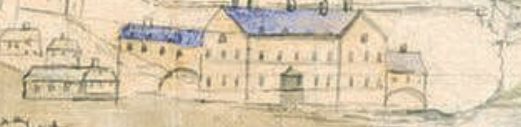

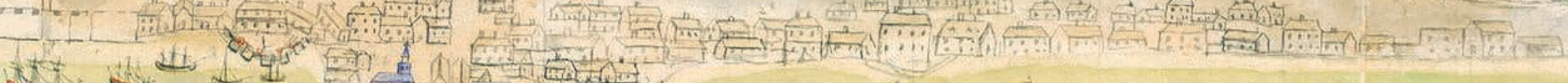

(4) Carre -igurative dupromt secours

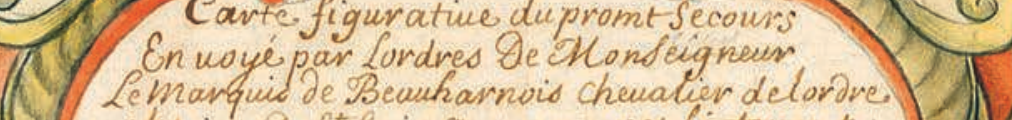

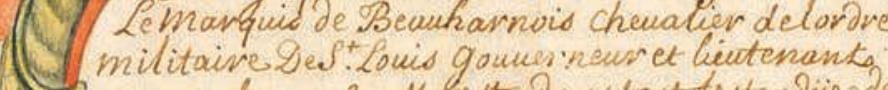

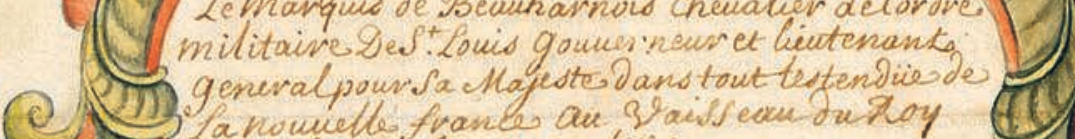

(1) 19

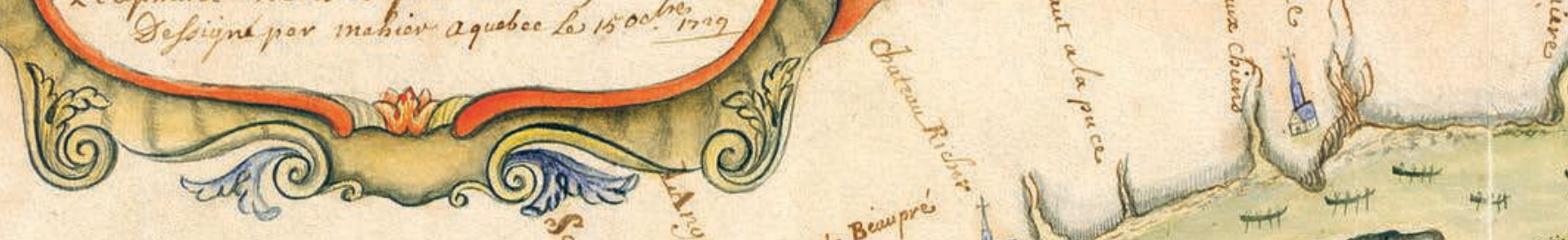

$+\frac{1}{4}+\frac{1}{2}$

(9)

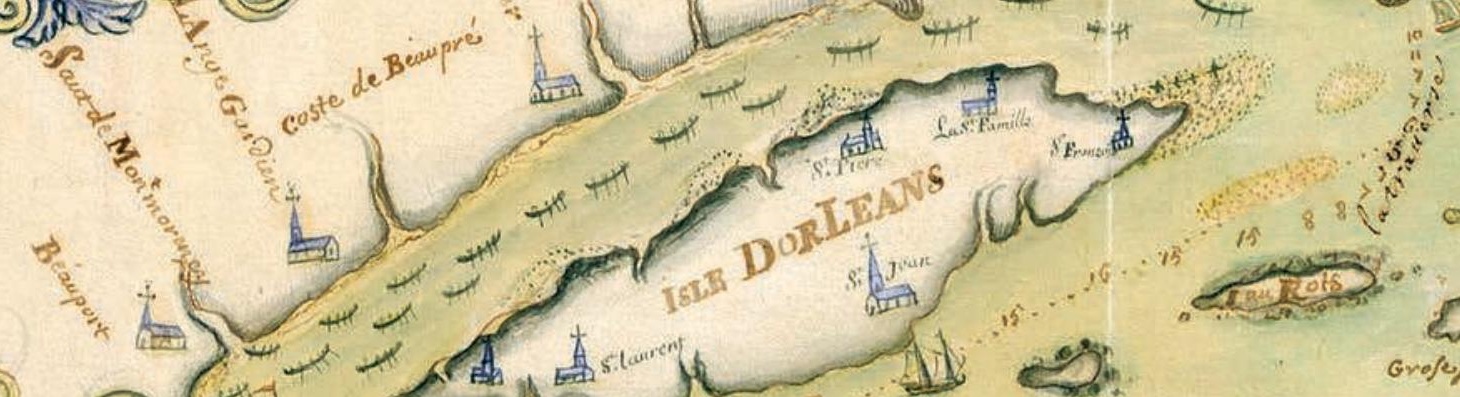

एम

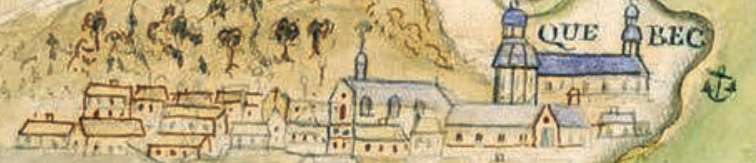
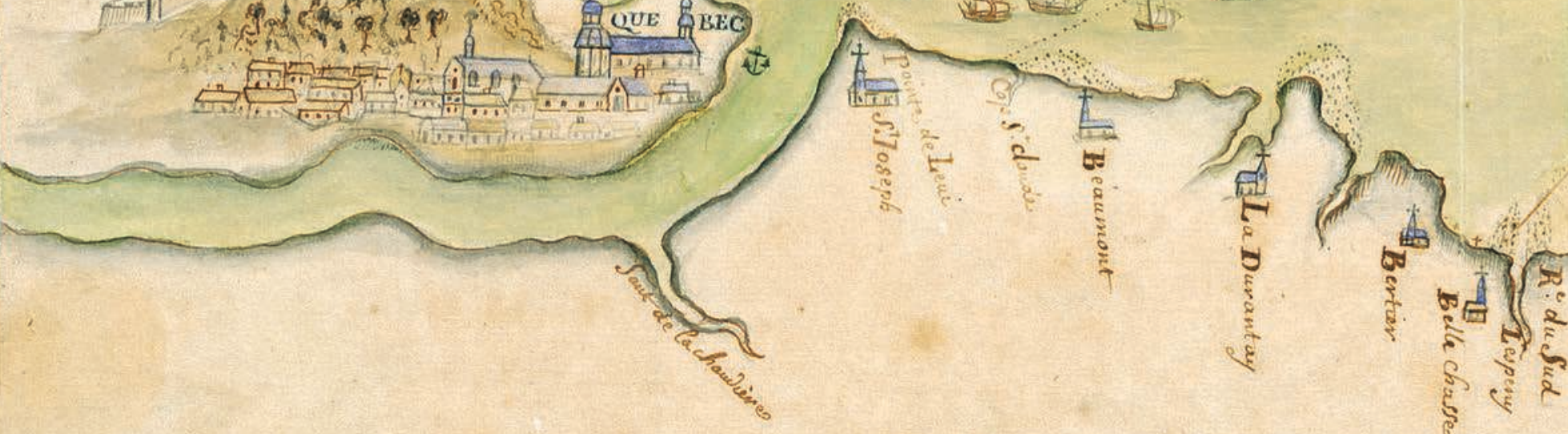




fig. 3. Charles Mahier. Carte figurative du promt secours envoyé par l'ordres [sic] de Monseigneur le Mr de Beauharnois,...gouverneur et lieutenant général pour Sa Majesté dans toutl'estendue de la Nouvelle France, au vaisseau du Roy I'Eléphant le 2 Septembre 1729 . Dessiné I'Eléphant, le 2 Septembre 1729. Dessine Papini, a Québec, lalle $56 \times 39 \mathrm{~cm}$. Paris, BnF, Cartes et Plans, GED-7825 (RES). 
19. Deuxième guerre intercoloniale ou Queen Anne's War (Laramie 2021)

20. Les mollusques employés dans la fabrication du wampum se développentexclusiven se developpentexclusiv te atlantique septentrionale près de Cape Cod, région colonisée par les Anglais. Ces derniers monopolisent de fait, depuis la seconde moitié du xvII siècle, la manufacture des perles par la soumission des nations par la soumission des
côtières (Ceci 1989).

21. Les Européens introduisen les cauris dans le nord-est de I'Amérique du Nord au xvI siècle, dans l'espoir probable d'établir dans l'espoir probable d'établir
un système de valeurs similaire à celui utilisé à l'époque en Afrique, mais ceux-ci ne sont pas bien acceptés en contexte amérindien (Ceci1989: 71 et73; Stolle 2016: 19).

22. Dénommée la «French and Indian War» dans le monde anglo-saxon.

23. Ces chiffres sont néanmoin à relativiser car la corruption régnait en Nouvelle-France, comme le soulignent les Mémoires publiés à titre posthume de Pierre Pouchot officier de marine français en poste au Canada de 1754 à 1761 (Pouchot 1781: 60-66). Quoi qu'il en soit, ces chiffres restent très élevés: la moitié des perles citées pouvaità elle seule permettre la production étant donné qu'une moyenne de deux mille perles (onze rangées de perles sur une longueur de deux centimètres) était nécessaire à la fabrication d'un collier d'après les sources de l'époque (Stolle 2016:103 sq.).

24. Les différents modèles de colonisation sont plus complexes et polymorphes qu'on ne l'a souvent écrit. Les colonies françaises notamment n'étaient ni homogènes ni semblables: le Canada et I'Acadie différaient par exemple de la Louisiane dans le type de relations établies avec les nations amérindiennes et la diffusion de l'esclavage (Jaenen 2001; Delâge 2006) à vingt-cinq ǵrains par jour); " [1]a lime et les forêts les mieux acierées ne peuvent travailler a percer ce coquillage [Mercenaria mercenaria] qu'avec bien de la peine»; bref, il faut «une patience extréme pour travailler les grains de Porcelaine tant blancs que bleus.» (BnF NAF 2550, Anonyme s. d. [vers 1725] : fos 25-27)

Le rapport souligne que les Iroquois, «sans contestation les plus habiles de tous ceux de l'Amérique Septentrionale», «font des amas de coliers pour la nation ce qui leur donne le moyen de faire reussir leurs affaires pendant que les autres nations qui n'ont point tant de prevoyance qu'eux sont souvent hors d'état de pouvoir traiter avec personne faute de coliers » (ibid. : fo 24).

La rédaction de ce mémoire vers 1726 témoigne tant de la centralité du wampum dans les affaires européennes et nord-amérindiennes que de l'absolue nécessité qu'il y a à s'en procurer. Le succès français que représente la paix de Montréal de 1701 a été suivi par l'entrée en guerre avec les colonies anǵlaises, dans le cadre de la guerre de Succession d'Espaǵne entre 1702 et $1713^{19}$. L'accès au wampum, obtenu par voie commerciale avec les Anglais, est rendu précaire et la disponibilité en perles et en colliers s'en trouve de toute évidence impactée ${ }^{20}$. Même s'il n'est rédigé que dix ans après, ce mémoire et la démultiplication des démarches mises en œuvre dans les dernières années de gouvernance du marquis de Vaudreuil (1703-1725) ainsi que celles de son successeur le marquis de Beauharnois (1726-1747) montrent bien qu'il est urgent pour la survie de la colonie de trouver une solution à cette dépendance. Beauharnois lance un cri d'alarme en novembre 1728 :

\section{La disette des Colliers de Porcelaine dont on se sert en ce païs-ci pour parler d'af- faires avec les sauvages en devenüe si grande et parmy eux et parmy nous, qu'il n'en reste plus dans les magasins des Roy, qu'on en oblige de faire refaire les Colliers qu'ils apportens, pour les degui- ser l orsqu'on est obligé a leur parler avec des Colliers. \\ (Beauharnois 1728)}

Les Français se tournent vers les escargots cauris, introduits sans succès en Amérique du Nord 21, ainsi que vers les perles de verre de différents types (Desjardins et Duguay 1992: 54 sq.). Beauharnois charge la Manufacture de Saint-Cloud de créer des perles en porcelaine imitant le wampum (Beauharnois 1728), puis semble peu convaincu, en 1729, par l'idée d'en faire réaliser en marbre (Beauharnois 1729).

Pendant la ǵuerre de Sept Ans ${ }^{22}$, pour se prémunir et être en capacité de dialoguer avec les nations amérindiennes et de négocier les alliances, le roi fait acheter aux commerçants canadiens Varin et Martel quatre millions de «ǵrains» (perles de wampum) entre 1755 et 1757 , payés dix livres par milliers de perles, soit quarante millions de livres (Estèbe 1763) ${ }^{23}$, une somme conséquente.
TISSER DES PERLES ET DES MOTS

UNE EMPREINTE FRANCAAISE DURABLE EN AMÉRIQUE DU NORD

La France adopte une politique différente de celle de l'Anǵleterre: ses colons ne s'installent ni ne cultivent la terre de manière intensive, ce qui aurait logiquement donné lieu, comme c'est le cas en Nouvelle-Anǵleterre, à des tensions et conflits avec la population autochtone locale; au contraire, les Français développent la traite avec les nations amérindiennes, leur intérêt étant dévolu à l'élargissement de leur réseau d'échange (Havard 200724). Sur le plan religieux, des divergences dans la politique de colonisation entre les deux puissances européennes s'observent également. Les ordres catholiques et protestants, conscients de la valeur de la langue dans leur entreprise missionnaire, enregistrent et documentent les langues autochtones pour permettre la communication et la conversion. Mais si la lecture et l'écriture sont au cœur de la pratique protestante, celles-ci étant essentielles à la compréhension de la parole de Dieu telle qu'elle s'exprime dans la Bible (Silverman 2007), la pratique catholique se centre davantage sur le rite, les sacrements, la répétition et accorde une place plus importante aux représentations visuelles des sphères ecclésiastique et non humaine. En ce sens, cette dernière s'avère particulièrement performante en contexte nord-amérindien car elle offre des parallèles avec la praxis spirituelle locale, comme l'offrande, la relation avec des entités «surnaturelles», le langage rituel, l'emploi de regalia associés aux cérémonies, la rétribution des ritualistes (Feest 1998). Les missionnaires œuvrent à rendre le catholicisme intelligible et attractif pour les autochtones (Havard 2003: 681-695).

À compter du XVII siècle, l'arrivée des ordres catholiques romains, en particulier ceux des Jésuites et des Récollets fondés en France, conduit au développement de missions le long de la vallée du SaintLaurent: ces villages sont peuplés de communautés déplacées de leurs territoires ancestraux par les conflits, et trouvant refuge là où se développe la colonie française (Lozier 2018). L'action missionnaire est particulièrement forte auprès des Abénakis, des Hurons, des Mohawks et des Nipissing (Jaenen 1985: 9 sq.). L'usaǵe des wampums, bien établi parmi ces nations, s'adapte à ce contexte: les communautés autochtones converties au christianisme vont les utiliser comme offrandes dévotionnelles afin de s'attirer les faveurs des saints les plus puissants et du clergé français. C'est ainsi que voient le jour, dans la colonie française, les colliers de wampum «votifs» ou «latinisés» (Clair 2009: 169): produits par les locaux à l'instigation des missionnaires, ils sont pour la première fois ornés d'inscriptions en latin visuellement porteuses du message ou de la «parole» qui leur est associé(e). Ceux-ci s'inscrivent dans des réseaux spirituels et économiques jésuites, et témoignent de la permanence et de la vitalité de conceptions autochtones liées à l'oralité, à la parenté et à l'alliance (Clair 2009).

À la période coloniale, en particulier pendant la guerre dite du Roi Philip (1675-1676) qui voit les Wampanoaǵ et Narraganssett combattre les colons 


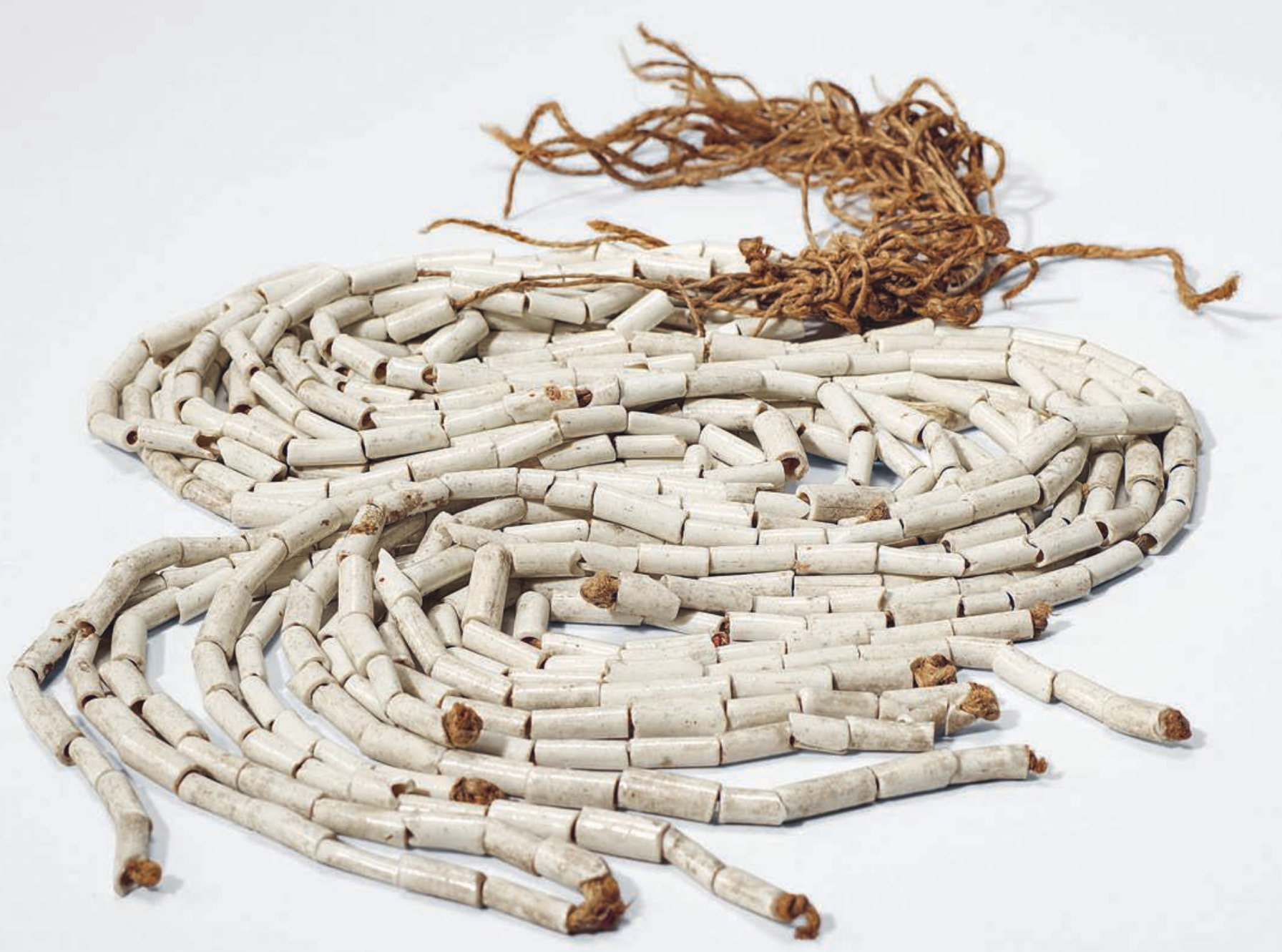

fig. 4. Ficelles en perles de «porcelaine» d'imitation de wampum

France (manufacture de Saint-Cloud ?), première moitié du xv॥e siècle. Terre cuite émaillée fibres végétales, L. $92 \mathrm{~cm}$, inv. 71.1878 .32 .267$.

Ancienne collection du Cabinet des médailles de la BnF ๑ musée du quai Branly-Jacques Chirac, photo Pauline Guyon. 
Introduction

fig. 5. Collier de wampum

Illinois (attribué à), Grand Lacs d'Amérique du Nord, avant 1725 Palourde (Mercenaria mercenaria), escargot de mer, cuir, fibres végétales, L. $83 \mathrm{~cm}$

inv. 71.1878.32.61. Ancienne collection du Cabinet des médailles, BnF @ musée du quai Branly-Jacques Chirac, photo Patrick Gries.

Ce collier est peut-être celui envoyé par Mamantouensa, chef des Kaskaskia à Louis XV via une délégation de quatre chefs des nations Illinois, Missouri, Osage et Otoe venue en France en 1725, accompagnée du père Beaubois, supérieur

des missions de la Compagnie de Jésus en Louisiane. La nation Kaskaskia appartient à la Confédération Illinois, composée de quatre «villages» dont les quatre figures d'hommes armés pourraient être des évocations.

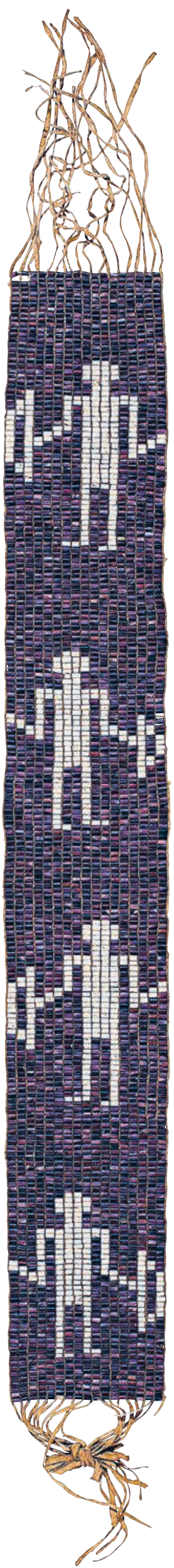


anǵlais et leurs alliés amérindiens de NouvelleAnǵleterre, plusieurs nations autochtones sont forcées de quitter leurs terres. Dans la seconde moitié du XVII ${ }^{\mathrm{e}}$ siècle, divers établissements missionnaires voient ainsi le jour en Nouvelle-France, en conséquence de la fuite massive d'anciens membres adoptifs de la Confédération iroquoise des Cinq Nations vers les colonies françaises du Canada. Ces réfugiés quittent la Confédération en quête d'une plus grande autonomie pour vivre selon leurs croyances et à l'abri de la domination iroquoise. Les missions les plus importantes, tels le village des Mohawks du Sault-Saint-Louis ${ }^{25}$ ou ceux des Abénakis de Bécancour et de Saint-François ${ }^{26}$, sont érigées le long du fleuve Saint-Laurent et de la rivière Champlain, voies de communication (et d'invasion) privilégiées entre les territoires sous domination française et anǵlaise. Il s'agit de villaǵes fortifiés jouant le rôle de remparts contre les incursions anglaises, qui représentent une réelle menace pour les établissements français, comme en témoigne le cas de la ville de Québec, siège administratif du Canada français, occupée à plusieurs reprises par les Anǵlais (Delâge et Sawaya 2001).

Les hommes de ces communautés ne se contentent pas de soutenir les Français dans leur lutte contre les Anǵlais. Ils jouent également un rôle actif dans le commerce lucratif de la traite pelletière. Ces nations copient la mode française, adoptant les vêtements taillés, comme les casaquins ${ }^{27}$ pour les femmes, et la croix chrétienne comme ornement. On les appelle les «Indiens domiciliés» (Brodhead 1855: 607, 609; Phillips 1998: 83 sq.), terme qui reflète leur conversion au christianisme, et les missionnaires se reposent sur eux pour convertir d'autres communautés autochtones. Quant aux femmes, elles sont à maints égards les garantes du maintien de la tradition car elles élèvent les enfants, leur enseignnent la lanǵue et initient les jeunes filles à l'artisanat. Pour leur production artisanale, elles ont recours à leur propre répertoire iconographique tout en introduisant de nouveaux motifs, suivant en cela les penchants et goûts individuels et collectifs. Il en est ainsi des colliers de wampum tissés par les Mohawks du Sault-SaintLouis : ils y intègrent le motif de la croix latine pour s'autodésiǵner, après que la France eut perdu ses colonies (Lainey 2004: 65; Stolle 2016: 168 et 200). La religion catholique sera, avec la lanǵue française, la principale empreinte laissée par la colonisation française dans le monde amérindien (voir l'entretien avec Nicole O'Bomsawin dans ce numéro).

À l'issue de la guerre de Sept Ans (1756-1763), la France perd toutes ses possessions en Amérique du Nord. Le traité de Paris (1763), qui met fin à la guerre et réconcilie la France, la Grande-Bretaǵne et l'Espagne après trois ans de négociation, consacre la cession des provinces de l'Acadie et du Canada à la GrandeBretagne, tandis que l'immense Louisiane est cédée à l'Espaǵne pour contrecarrer l'hégémonie anǵlaise. Malgré cette perte, les colons français continuent d'entretenir des relations amicales et économiques avec leurs anciens alliés autochtones, que ce soit par le biais des liens familiaux ou commerciaux. À Montréal, le cour commercial de l'ancienne colonie du Canada, les marchands canadiens-français contrôlent encore le lucratif commerce des fourrures, qui n'atteint son apogée qu'au XIX siècle. Ils s'appuient sur leurs anciens réseaux qui s'étendent à l'intérieur des terres le long des voies navigables vers le Missouri et le Mississippi (Havard 2019).

Dans la décennie de 1770, la France connaît un regain d'influence en Amérique du Nord dans le contexte de la guerre de l'Indépendance des États-Unis, lorsque la Couronne décide d'apporter son soutien aux treize colonies dans leur lutte contre son rival anǵlais.

La France royale apparaît représentée sur les colliers de wampum par le motif de la croix pattée, qui symbolise la mission principale qui lui est attribuée, à savoir la défense de l'Éǵlise catholique romaine. Cette fois cependant, cette croix chrétienne incarnant la participation française n'est plus figurée au centre mais à la périphérie des colliers, car elle ne possède plus de colonies en Amérique du Nord (Stolle 2016: 201).

\section{TISSER LES LIENS AUJOURD'HUI}

Pendant toute la période coloniale française en Amérique du Nord, plusieurs délégations de nations autochtones alliées sont envoyées à la cour de France afin d'attirer l'attention sur leur taille et leur importance. Celles-ci, composées le plus souvent d'un ou plusieurs chefs accompagnés de leur entourage, sont reçues par le roi. L'un des tout premiers délégués est Iwanchou, un célèbre chef de guerre Mi'kmaq qui, lors de son audience, dépose son bandeau de tête en wampum aux pieds de Louis XIV (Thwaites 1897 : 223). En retour, il reçoit pour lui et ses compaǵnons des vêtements ornés de fils d'or, des épées et divers autres insiǵnes de statut. La dernière délégation accueillie à la cour de France est celle des membres de la nation Iowa reçue par Louis-Philippe en 1845 (Anonyme 1845: 2). Cette rencontre témoigne du dernier échange de wampum entre la France et une nation amérindienne. Le wampum est ainsi omniprésent dans les relations établies entre les Français et les nations amérindiennes et suit les aléas de l'histoire coloniale française en Amérique du Nord. Dès le XvII ${ }^{\mathrm{e}}$ siècle, les perles en coquillage blanc confectionnées et utilisées en Amérique du Nord pendant des millénaires acquièrent au contact des Européens une taille standardisée et une fonction renouvelée. Les Français jouent un rôle important dans les échanges de perles et dans l'avènement du collier de wampum au début de la période de contact en Nouvelle-France (voir Laurier Turgeon dans ce numéro). Les représentants du roi de France tissent avec les nations amérindiennes du Canada des relations diplomatiques et commerciales durables (voir Gilles Harvard dans ce numéro). En témoigne l'important corpus de «paroles» françaises et amérindiennes, transcrites et conservées dans les archives coloniales, et l'ensemble de colliers, de branches et d'objets en wampum encore aujourd'hui conservés en France. Ces pièces qui datent de la période d'Ancien Régime sont les témoins des alliances durables entretenues jadis entre Amérindiens et Français.
25. Aujourd'hui Kahnawake.

26. Aujourd'hui Wôlinak et Odanak respectivement.

27. C'est le terme désignant une veste courte pour femme devenu obsolète au xvIII ${ }^{\mathrm{e}}$ siècle (Chambaud et Robinet 1776: 126). 
28. D'autres collections de référence sont celles conservées à I'American Museum of Natural History de New York, au musée McCord de Montréal, ainsi qu'au National Museum of the American Indian et au National American Indian et au Nation à Washington, D. C.

29. Michael Galban, conseille scientifique de l'exposition. Propos tenus lors d'une consultation virtuelle des consultation virtuelle des du quai Branly-Jacques Chirac le 3 décembre 2020 .
Ces artefacts ont été amenés en France et intégrés aux collections royales, aristocratiques, ecclésiastiques et à celles du milieu savant, avant d'être confisqués au moment de la Révolution française pour rejoindre les collections nationales. À l'exception du Haudenosaunee Council (Conseil des Six Nations iroquoises) qui a rapatrié près d'une quarantaine de wampums dans les années 1988-1997, peu de collections au monde disposent d'un ensemble de spécimens aussi ancien, qui plus est avec une telle variété typologique (voir Paz Núñez-Regueiro et Nikolaus Stolle dans ce numéro) ${ }^{28}$. Au fil de leurs pèlerinaǵes à travers les institutions qui partaǵent cet héritage, les objets de wampum témoiǵnent non seulement des cultures amérindiennes mais aussi des cultures européennes. Aujourd'hui, ils trouvent leur place dans les vitrines des musées européens et dans les cérémonies amérindiennes, dans les livres d'histoire et dans les mains d'artistes autochtones, et même devant les tribunaux en tant que «preuve juridique» (Borrows 2016: 7, 10, 27 ; Nichols 2016: 10 sq., 119 sq.; Schulte-Tenckhoff 1998: 247, 270 ; Dickson-Gilmore 1995 : 150, 226, 252-261, 330, 432-444).

Les wampums sont des objets mémoriels qui agissent comme témoins d'une époque. Ils rappellent les relations issues de la rencontre de deux mondes et, de ce fait, ils stimulent le questionnement et la réflexion sur les traditions diplomatiques autochtones, l'adaptation européenne à ces traditions, la mythologie iroquoienne et le monde amérindien aux XVII ${ }^{\mathrm{e}}$-XVIII ${ }^{\mathrm{e}}$ siècles. Côté amérindien, les groupes ont recours à des outils mnémoniques et insèrent des motifs géométriques et figuratifs dans leurs colliers de wampum, pour enregistrer dans la matière une partie de leur signification (Druke 1995: 89, 93; Fenton 1998: 180, 185, 333, 512; Murray 2000: 125, $130,134)$. Ces motifs sont étroitement associés à la pictographie guerrière, documentée en Amérique du Nord sur d'autres supports avant le développement des colliers tissés (voir Nikolaus Stolle dans ce numéro). Les sources relatives au wampum ont souvent été exploitées sans tenir suffisamment compte des spécificités des sociétés algonquiennes ou iroquoiennes auxquelles elles se référaient, alors qu'une grande diversité caractérisait le contexte historique et culturel des différentes nations de la région. Ainsi, les cas des Hurons et des Iroquois sont souvent étudiés sans aucune distinction alors que leur culture n'est pas identique. De même, les Algonquins ont souvent été délaissés dans les études sur le wampum à l'époque moderne, alors que ce sont eux qui ont introduit la manufacture de perles en coquillage blanc dans leur territoire originel sur la côte atlantique.

L'érosion de l'autonomie amérindienne sonne le ǵlas de l'acceptation partagée du wampum en Amérique du Nord, dont l'usage est alors restreint à la sphère autochtone, aux affaires internes et intertribales jusqu'à la seconde moitié du XIX ${ }^{\mathrm{e}}$ siècle. Les archives autochtones, dont les wampums font partie, sont ensuite dispersées : les colliers et les ficelles ou branches sont vendues et rejoignent des collections publiques ou privées (voir Jonathan Lainey dans ce numéro). Avec les mouvements d'autodétermination menés par les Premières Nations, les colliers de wampum sont aujourd'hui devenus des symboles de l'autonomie autochtone et publiquement reconnus pour certains d'entre eux, notamment sur les drapeaux nationaux. Le wampum joue donc de nouveau un rôle majeur parmi les communautés nord-amérindiennes (voir Michael Galban, Jamie Jacobs et Peter Jamison dans ce numéro).

C'est en raison de la valeur universelle du wampum et de son rôle clé dans les échanǵes et relations entre nations et individus que l'exposition «Wampum Perles de diplomatie en Nouvelle-France» (8 février15 mai 2022) a vu le jour au musée du quai BranlyJacques Chirac. La recherche menée pour cette manifestation s'inscrit dans le cadre du projet de recherche CRoyAN (Collections royales d'Amérique du Nord) lancé par le musée en collaboration avec plusieurs partenaires français et nord-amérindiens. Celui-ci vise à examiner l'importante collection d'objets collectés entre 1650 et 1850 dans les territoires actuels du Canada et des États-Unis. L'étude des wampums conservés en France dans le cadre de cette exposition implique des chercheurs et porteurs culturels aux profils disciplinaires variés, issus des nations européennes et amérindiennes engagées sur l'échiquier politique du nord-est de l'Amérique du Nord aux temps de la Nouvelle-France: en sus de l'équipe travaillant en France, les partenaires et conseillers scientifiques autochtones du projet d'exposition sont membres des nations Haudenosaunee, HuronneWendate et Abénaki.

Les dialogues noués autour des objets rendent compte des sentiments contrastés que suscite l'étude des wampums conservés en France: en effet, si ceux des collections muséales sont le plus souvent issus de fouilles archéologiques et de contextes funéraires, ou de modalités d'acquisition associées à un sentiment de spoliation historique, les pièces arrivées en France par le biais d'échanges et d'accords avec les partenaires amérindiens témoiǵnent, quant à elles, de la souveraineté des nations autochtones, de la conversion à la religín catholique et de l'ancienneté des relations entre Français et Nord-Amérindiens. Ce sont des objets aux valeurs multiples que la perception contemporaine rend ambivalents et profondément métisses. Ainsi, face à un collier de wampum «votif», dont la production souliǵne l'emprise missionnaire dans la réǵion, l'un de nos partenaires exprimait récemment:

Ce collier est vraiment intéressant car, d'un point de vue autochtone, il répond au format et aux matériaux les plus appréciés, les plus sacrés et puissants. Et comme il s'agit, en plus, d'un collier dévotionnel dans les mains des jésuites, il a probablement été béni par les jésuites, consacré, et il devient ainsi un emblème de sacralité pour les deux peuples, il devient d'une certaine manière doublement sacré 29 . 
Les échanges autour de ces pièces rendent aussi compte du «déséquilibre d'autorité » présent dans les échanges, qui se reflète à la fois dans la prééminence d'un savoir académique construit souvent loin du terrain et sur la base de sources partielles et partiales, vis-à-vis des connaissances dont sont dépositaires les porteurs culturels autochtones, et dans la place prioritaire accordée aux sources écrites au détriment des sources orales provenant d'un terrain ethnographique ou issues d'une tradition orale transmise à travers les générations. La recherche menée sur le wampum dans le cadre des articles qui suivent montre qu'on ne peut pas situer dans le temps et l'espace tout ce qui se trouve dans la pensée autochtone et dans le bagage du savoir accumulé depuis des siècles. Il nous faut comprendre et accepter que ces connaissances émanent de la compréhension amérindienne des événements passés, ainsi que le résumait récemment un autre de nos partenaires ${ }^{30}$. La collecte européenne, un phénomène unique au monde, entraîne une responsabilité de partage de cette richesse de savoir humain. Les collections d'Ancien Régime aujourd'hui conservées en France offrent l'opportunité d'élargiir de nouvelles et d'anciennes relations, suivant l'usage fait du wampum depuis son apparition.
30. Jonathan Lainey, conseiller scientifique de l'exposition. Propos échangés le 2 juin 2021.

Musée du quai Branly-Jacques Chirac

Paz.NUNEZ-REGUEIRO@quaibranly.fr Nikolaus.STOLLE@quaibranly.fr 


\section{Archives}

\section{Académie des Sciences}

1979 Procès-verbaux

de l'Académie des Sciences (1795-1835). Paris, Imprimerie nationale.

\section{Bibliothèque nationale de France, Département} des manuscrits, NAF 2550:

Recueil de pièces diverses, la plupart relatives à l'histoire de la première moitié du règne de Louis XV. V-VIII Marine et Colonies (1667-1735): - Anonyme, s. d. [vers 1725] «Mémoire concernant les coliers de porcelaine des Sauvages, leurs différents usages et la matière dont ils sont composés », fos 24-27 (voir reproduction dans ee numéro, p. 146).

- Anonyme, s. d. [1726]

"Paroles des sauvages Iroquois a M. le Marquis de Beauharnois...», fos $36-41$ (voir reproduction dans ce numéro, p. 151).

\section{Bibliographie}

\section{Anonyme}

1845 «Visite des indiens Ioway au roi », Le Constitutionnel, mereredi 23 avril.

\section{Bacqueville de La Potherie Claude-Charles Le Roy (de)}

1722 Histoire de l'Amérique septentrionale. Paris, Nion et Didot.

\section{Beauharnois, Charles (de)}

1728 «Lettre de Beauharnois concernant la pénurie de collier de porcelaine», Québec, 8 novembre. Bibliothèque et archives du Canada, Fonds des colonies MG1, C11A (Correspondance générale, Canada), microfilm : F-50.

1729 «Lettre de M. de Beauharnois au Ministre», 25 octobre. Archives nationales du Canada MG8, A1, t. XI : 2478-2479, cité dans Lainey 2004: 14.

\section{Beaulieu, Alain \\ et Viau, Roland}

2001 La Grande Paix: chromique d'une saga diplomatique.

Montréal, Libre Expression Itée.

Beaupré, Andrew R.

2021 " "The Jesuit mission proves we were here": The Case of Eigghteenth-Century Jesuit Mission Aiding Twenty-First Century Tribal Recognition », Journa of Jesuit Studies 8: 454-473.

\section{Black, Mary B.}

1977 «Ojibwa Power Belief System», in Raymond D. Fogelson et Richard N. Adams (dir.), The Anthropology of power. New York/San Francisco/Londres Academic Press : 141-151.

\section{Borrows, John}

2016 «Outsider Education Indigenous Law and Land-based Learning », Windsor Yearbook of Access to Justice 33 (1): 1-27.

\section{Brodhead, John Romeyn}

1855 Documents Relative to the Colonial History of the State of New York: Procured in Holland England, and France (compilation et édition par $E$.

B. O'Callaghan), t. IX. New

York, Weed and Parsons.

\section{Callender, Charles}

1978 «Illinois», in William C.

Sturtevant (dir.), Handbook

of the North American Indians.

Northeast, t. $X V$. Washington,

D. C., Smithsonian Institution

673-680.

Chambaud, Louis et Robine, Jean-Baptiste-René

1776 Nouveau Dictionnaire

François-Anǵlois, et Anǵlois-

François, t I. Paris/Amsterdam,

Théophile Barrois jeune/

Arkstée \& Merkus.

Champlain, Samuel (de)

1603 Des sauvages, ou Voyage de Samuel Champlain, de

Brouage, fait en la France

nouvelle l'an mil six cens trois.

Paris, Claude de Monstrœil.

\section{Ceci, Lynn}

1982 «The Value of Wampum among the New York Iroquois: A Case Study in Artefact Analysis », Journal of Anthropological Research 39: 97-107.

1989 «Tracinǵ Wampum's Oriǵins: Shell Bead Evidence from Archaeological Sites in Western and Coastal New York», in Charles F. Heyes III et Lynn Ceci (dir.) Proceedings of the 1986 Shell Bead Conference. Rochester/ New York, Rochester Museum and Science Center: 63-80.

1993 «Native Wampum as a Resource in the SeventeenthCentury World System», in Laurence M. Hauptman et James D. Wherry (dir.) et James D. Wherry (dir.),
The Pequots in Southern New England: The Fall and Rise of an American Indian Nation. Norman/Londres, University of Oklahoma Press : 48-63.

\section{Clair, Muriel}

2009 «Notre-Dame de Foy en Nouvelle-France (1669-1675): histoire des statuettes de Foy et des wampum des Hurons chrétiens », Annales de la Société archéologique de Namur. 167-192.

\section{Cruzat, Francisco}

1909 [1777] «Lettre du 26 novembre 1777 , San Luis de Ylinoeses », in Louis Houck (dir.), The Spanish regime in Missouri..., t I. Chicaǵo, R. R. Donnelley \& Sons Company: 135.
Dekoninck, Ralph

"Propagati Imaginum: The

Translated Images of Our Lady

of Foy », in Christine Göttler

et Mia M. Mochizuki (dir.), The Nomadic Object: The Challenge of World for Early Modern

Religious Art. Leiden/Boston, Brill : 241-267.

\section{Delâge, Denys}

2006 «Modèles coloniaux métaphores familiales et changements de régime en Amérique du Nord, $\mathrm{XVII}^{\mathrm{e}}-\mathrm{XIX}^{\mathrm{e}}$ siècles », Traces et itinéraires 60 : 19-78.

\section{Delâge, Denys et Sawaya} Jean-Pierre

2001 «Les oriǵines de la Fédération des Sept Feux», Recherches amérindiennes au Québec 31 (2) : 43-54.

DeMallie, Raymond J.

2001 Handbook of North American Indians, t. XIII Plains. Washinǵton, Smithsonian Institution.

\section{Desjardins, Paulins} et Duguay, Geneviève 1992 Pointe-à-Callière: l'aventure Montréalaise. Montréal, Septentrion.

\section{Dickson-Gilmore,} Elizabeth Jane

1995 «Resurrecting the Peace: Separate Justice and the Invention of Legal Tradition in the Kahnawake Mohawk Nation », thèse de doctorat, The London School of Economics and Political Science.

Druke, Mary A.

1995 [1985] «Iroquois Treaties Common Forms, Varyino Interpretations », in Francis Jennings (éd.) The History and Culture of Iroquois Diplomacy. An Interdisciplinary Guide to the Treaties of the Six Nations and Their League. New York, Syracuse University Press: 85-98.

\section{Estèbe, Guillaume}

1763 «Memoire pour Guillaume Estebe, ecuyer, secretaire du Roi, près la Cour des aydes de Bordeaux, conseiller honoraire au conseil supérieur de Quebec... 16 sq. Paris, De l'imprimerie de Cl. Hérissant, rue neuve Notre-Dame.

\section{Feest, Christian}

1998 Beseelte Welten. Die Religionen der Indianer Nordamerikas 9. Freiburg/ Bâle/Vienne, Herder.

Fenton, William N.

1998 The Great Law and the Longhouse: A Political History of the Iroquois Confederacy.

Norman, University of Oklahoma Press.

\section{Foster, Michael K.}

1996 «Language and the Culture History of North America », in Ives Goddard (dir.), Handbook of the North American Indians, t. XVII : Languages. Washinǵton D. C, Smithsonian Institution: 64-110.

Gagnon, François Marc

1975 La Conversion par l'image: un aspect de la mission

des jésuites auprès des Indien du Canada au XVII siècle. Montréal, Bellarmin.

Hale, Horatio

1881 Hiawatha and the Iroquois Confederation: A Study in

Anthropology. Salem (Mass.)

Salem Press.

\section{Havard, Gilles}

2003 Empire et métissages: Indiens et Français dans le Pays d'en Haut (1660-1715). Paris, Presses de l'université Panthéon-Sorbonne.

2007 «La France en Amérique du Nord», in Christian Feest (dir.), Premières Nations, collections royales: les Indiens des forêts et des prairies d'Amérique du Nord. Paris,

Réunion des musées nationaux et musée du quai Branly 19-26.

2019 L'Amérique fantôme : les aventuriers francophones du Nouveau Monde. Paris, Flammarion.

\section{Havard, Gilles \\ et Vidal, Cécile}

2019 [2003] Histoire

de l'Amérique française. Paris, Flammarion.

Hazard, Samuel (dir.)

1838-1853 Minutes of the Provincial Council of Pennsylvania from the Organization to the Termination of the Proprietary Government, March 10, 1683 to September 
1781 Mémoire sur la dernière guerre de l'Amérique septentrionale, entre la France et

Schulte-Tenckhoff, Isabelle 1998 «Reassessing the Paradiǵm of Domestication: The Problematic of Indigenous Treaties», Revu d'études constitutionnelles 4 (2) : 239-289

Silverman, David J.

2007 Faith and Boundaries: Colonists, Christianity, and Community among the Wampanoag Indians of Martha's Vineyard, 1600-1871. Cambridge University Press.

\section{Silverstein, Michae}

1996 «Dynamies of Linǵuisite

Contact», in Ives Goddard

(dir.), Handbook of the North

American Indians, t. XVII

Languages. Washington D. C.,

117-136.

Stolle, Nikolaus

2016 Talking Beads: The Histor

of Wampum as a Value and

Knowledge Bearer, From It

Very First Beginnings Until

Today. Hamburǵ, Dr. Kovač.

Turgeon, Laurier

2001 «French Beads in France and Northeastern North America During the Sixteenth Century »,

Historical Archaeology 35 (4)

$58-8$

Thwaites, Reuben Gold (éd.)

1897 The Jesuit Relations and Allied Documents: Travels and Explorations of the Jesuit Missionaries in New France, 1610-1791, t. XXV. Cleveland, The Burrow Brothers.

\section{Vachon, André}

1970 «Colliers et ceintures

de porcelaine chez les Indiens

de la Nouvelle-France

Les Cahiers des dix 35, 1970 251-278.

Wray, Charles F.

2003 Wampum and Glass

Bead Belts of the Seneca

Iroquois, Richard D. Hamell

(éd.). Rochester, New York.

Ci-contre et ouverture

Collier de wampum Illinois (attribué à), Grand Lacs d'Amérique du Nord, avant1725. Palourde (Mercenaria mercenaria) escargot de mer, cuir, fibres végétales, L. $83 \mathrm{~cm}$ Ancienne collection du Cabinet des Branly-Jacques Chirac photo Patrick Gries (détail).

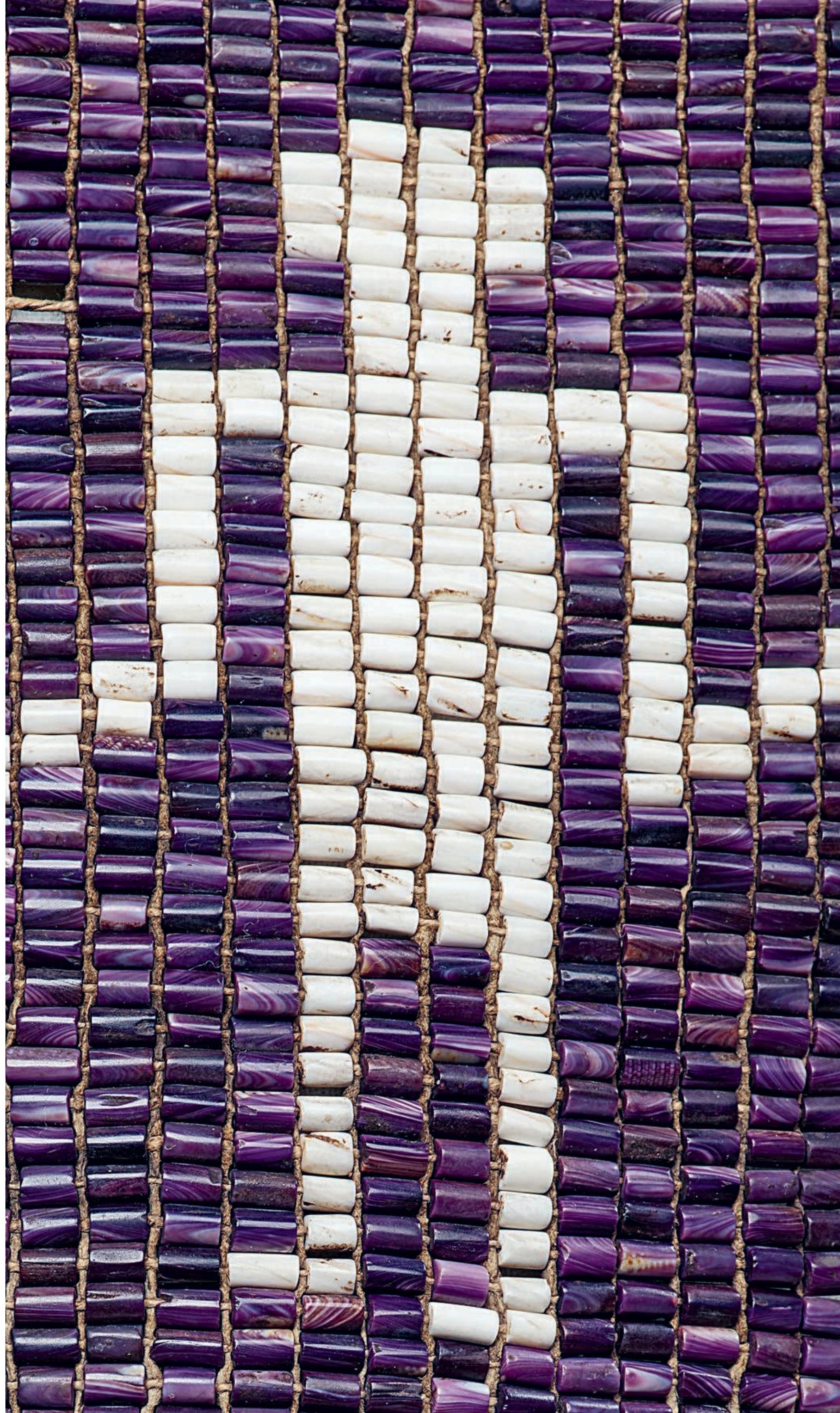

